\title{
Trajetória de Mulheres Rastreadas para o Câncer de Mama na Rede Pública de Saúde
}

https://doi.org/10.32635/2176-9745.RBC.2018v64n4.200

Trajectory of Breast Cancer Screened Women in Unified Health System

Trayectoria de Mujeres Rastreadas para el Cáncer de Mama en la Red Pública de Salud

Jeane Glaucia Tomazelli'; Isabel dos-Santos-Silva ${ }^{2}$; Gulnar Azevedo e Silva ${ }^{3}$

Resumo

Introduçáo: Conhecer os tempos entre as etapas do programa de rastreamento é importante para acompanhar as açôes de controle de câncer. Objetivo: Estimar o intervalo de tempo entre o resultado suspeito de malignidade pela mamografia e o início do primeiro tratamento, e identificar fatores associados ao seu início, entre mulheres rastreadas para câncer de mama, nos serviços do Sistema Único de Saúde (SUS) do município do Rio de Janeiro. Método: Registros do Sistema de Informação do Controle do Câncer de Mama para mulheres de 40-69 anos, com uma mamografia de rastreamento efetuada em julho-dezembro/2010, com resultados suspeitos (BI-RADS ${ }^{\bullet}$ 4 ou 5), foram relacionados com os Sistemas de Informação Hospitalar, Ambulatorial e de Mortalidade para 2010-2012. O tempo foi estimado pelo método de Kaplan-Meier, e seus determinantes identificados pela regressão de Cox. Resultados: Entre 158 mulheres com mamografia alterada, foram identificados registros de 66 (41,8\%) casos de câncer de mama. Destes, 12,1\% tinham informações sobre biópsias prévias. O tempo mediano entre a mamografia e o início do tratamento foi de 206 dias, sendo menor para mulheres entre $40-49$ anos (138 dias) do que para as mais idosas (190 para mulheres de 50-59 anos; 234 dias para mulheres de 60-69 anos) (Log-rank, p<0,05). Mulheres que repetiram mamografia apresentaram maior atraso (hazard ratio: 0,36; intervalo de confiança de 95\% 0,19-0,72). Conclusáo: Há poucas biópsias registradas no SUS e longo tempo até o início de tratamento, mesmo quando as mamografias são solicitadas por hospitais especializados, demonstrando necessidade de o SUS melhorar o seguimento de mulheres com mamografia suspeita.

Palavras-chave: Programas de Rastreamento; Neoplasias da Mama; Tempo para o Tratamento; Detecção Precoce de Câncer; Sistemas de Informação em Saúde.

\begin{abstract}
Introduction: Knowing the times between the steps of a screening program is important to track the cancer control actions. Objective: Estimate the time interval between the suspected result of malignant mammography and the beginning of the first treatment, and to identify associated factors with its onset, among women screened for breast cancer, in services of the Unified Health System (SUS) in the city of Rio de Janeiro. Method: The records of the Information System of Breast Cancer Control for women aged 40-69 years, with a screening mammography carried out in july-december/2010, and whose results revealed suspicious (BI-RADS 4 or 5 ) were related to Hospital, Outpatient and Mortality Information Systems for 2010-12. The time was estimated by Kaplan-Meier method, and its determinants were identified through Cox regression. Results: 158 women with altered mammography, records were identified, with breast cancer diagnosis, in the other databases for $66(41.8 \%)$. Of these, $12.1 \%$ had information on biopsies. The median time between mammography and the start of treatment was 206 days, being lower for women aged $40-49$ years ( 138 days) than for older women (190 for women aged 50-59; 234 days for women of 60-69 years) (Log rank, $\mathrm{p}<0.05$ ). Women who repeated the mammography (hazard ratio: 0.36 ; $95 \%$ confidence interval $0.19-0.72$ ) presented a longer time. Conclusion: There are few biopsies registered in the SUS and long time until the beginning of treatment, even when mammographies are requested by specialized hospitals, which demonstrates the need for SUS to improve the follow-up of women with suspected mammography.

Key words: Mass Screening; Breast Neoplasms; Time-to-Treatment; Early Detection of Cancer; Health Information Systems.
\end{abstract}

\section{Resumen}

Introducción: Conocer los tiempos entre las etapas de programa de rastreo es importante para controlar las acciones de control del cáncer. Objetivo: Estimar el intervalo de tiempo entre resultado sospechoso de malignidad de la mamografía y inicio del primer tratamiento, y identificar factores asociados, entre mujeres rastreadas para cáncer de mama, en servicios del Sistema Único de Salud (SUS), municipio de Río de Janeiro. Método: Registros del Sistema de Información del Control del Cáncer de Mama para mujeres de 40-69 ańos, con una mamografía de rastreo efectuada en julio-diciembre/2010, cuyos resultados revelaron alteraciones sospechosas (BI-RADS ${ }^{\circledR} 4$ o 5) relacionados con Sistemas de Información Hospitalaria, Ambulatorial y de Mortalidad para 2010-12. El tiempo fue estimado pelo método Kaplan-Meier, y sus determinantes identificados por la regresión de Cox. Resultados: Entre 158 mujeres con mamografía alterada, fueron identificados registros, con diagnóstico de cáncer de mama, en otras bases para $66(41,8 \%)$. Dellas, $12,1 \%$ tenía informaciones sobre biopsias. Tiempo medio entre la mamografía y inicio del tratamiento fue de 206 días, siendo menor para mujeres entre 40-49 ańos (138 días) que para ancianas ( 190 para mujeres de 50-59 ańos, 234 días para mujeres de 60-a 69 años) (Log-rank, $\mathrm{p}<0,05$ ). Mujeres que repitió la mamografía (hazard ratio: 0,36 , intervalo de confianza del 95\% 0,19-0,72) presentaron mayor tiempo. Conclusión: Hay pocas biopsias registradas en SUS y largo tiempo hasta el inicio del tratamiento, incluso cuando las mamografías son solicitadas por hospitales especializados, demostrando la necesidad del SUS de mejorar el seguimiento de mujeres con mamografía sospechosa

Palabras clave: Tamizaje Masivo; Neoplasias de la Mama; Tiempo de Tratamiento; Detección Precóz del Cáncer; Sistemas de Información en Salud.

\footnotetext{
${ }^{1}$ Instituto Nacional de Câncer José Alencar Gomes da Silva (INCA). Rio de Janeiro (RJ), Brasil. Orcid iD: https://orcid.org/0000-0002-2472-3444

${ }^{2}$ School of Hygiene \& Tropical Medicine, Londres. Orcid iD: https://orcid.org/0000-0002-6596-8798

${ }^{3}$ Universidade do Estado do Rio de Janeiro (Uerj). Rio de Janeiro (RJ), Brasil. Orcid iD: https://orcid.org/0000-0001-8734-2799

Endereço para correspondência: Jeane Glaucia Tomazelli. Rua Marquês de Pombal, 125, 70 andar - Centro. Rio de Janeiro (RJ), Brasil. CEP $22230-240$.

E-mail: jtomazelli@inca.gov.br
} 


\section{INTRODUÇÃO}

No Brasil, como em outras regiôes do mundo, a incidência de câncer de mama é maior nas regióes mais desenvolvidas do país se comparadas às menos desenvolvidas ${ }^{1}$, embora variaçóes regionais possam refletir diferenças na capacidade diagnóstica dos serviços de saúde $^{2}$. Apesar de ser o tipo de câncer com a mais alta mortalidade ${ }^{3}$ entre as brasileiras, recentemente vem se observando queda nas capitais das Regiôes Sudeste e Sul do país, possivelmente relacionada ao melhor acesso ao diagnóstico e ao tratamento nessas localidades ${ }^{4}$. Embora as taxas de incidência sejam inferiores à de países de alta renda, a razão mortalidade/incidência no Brasil é maior do que no Reino Unido, União Europeia e Estados Unidos, dada a alta letalidade ainda verificada no Brasil para essa neoplasia ${ }^{5}$, o que indica a necessidade de se investir na detecção precoce da doença e no seu tratamento ${ }^{6-8}$.

Em 2015, o município do Rio de Janeiro foi a capital com a mais alta taxa de mortalidade ajustada por idade pela populaçáo brasileira em $2010(19,59 / 100 \mathrm{mil})^{3}$. A rede pública de saúde do município do Rio de Janeiro se distribui por dez áreas programáticas (AP), com populaçóes de composiçóes etárias diferentes: um perfil mais jovem nas AP 4.0, 5.2 e 5.3; intermediário nas AP 3.1, 3.2, 3.3 e 5.1; e mais envelhecido nas AP 1.0, 2.1 e $2.2^{9,10}$, sendo verificada uma maior mortalidade por câncer de mama nas AP 2.1 e $2.2^{10,11}$.

O fluxo desde a realização da mamografia de rastreamento até o tratamento dos casos envolve todos os níveis de atenção ao cuidado à saúde. O Ministério da Saúde recomenda rastreamento mamográfico bienal para mulheres entre 50 e 69 anos de idade ${ }^{12}$. Conforme o resultado, baseado no sistema de classificação BI-RADS ${ }^{\oplus 13}$, a mulher pode ser orientada a retornar ao rastreamento em dois anos (BI-RADS ${ }^{\bullet} 1$ ou 2 ), repetir um novo exame de imagem (BI-RADS $\left.{ }^{\bullet} 0\right)$, realizar mamografias em intervalos semestral/anual (BI-RADS $\left.{ }^{\bullet} 3\right)$ ou investigação histopatológica (BI-RADS 4 ou 5). Caso o resultado indique a presença de neoplasia maligna, a mulher deve seguir para tratamento em um hospital especializado na alta complexidade.

Os tempos entre as etapas de realização da mamografia, resultado, investigação de uma lesão suspeita e início do tratamento são importantes para acompanhar o impacto das açôes de controle de câncer em diversos países ${ }^{14-17}$. No Brasil, o tempo entre diagnóstico e início de tratamento passa a ser acompanhado, a partir de 2013, tendo como meta o intervalo menor que $60 \mathrm{dias}^{18}$.

O objetivo deste estudo foi estimar o intervalo de tempo entre o resultado suspeito e altamente suspeito de malignidade (BI-RADS ${ }^{\oplus} 4$ ou 5 ) na mamografia de rastreamento e o início do tratamento, bem como analisar os fatores associados a esse intervalo, para mulheres residentes no município do Rio de Janeiro, assistidas nos serviços do Sistema Único de Saúde (SUS), localizados nesse município.

\section{MÉTODO}

Foram identificadas todas as mulheres entre 40 e 69 anos de idade residentes no município do Rio de Janeiro que tiveram resultado de mamografia de rastreamento com suspeita de malignidade (BI-RADS ${ }^{\circ} 4$ ou 5 ) nas informaçóes registradas no segundo semestre de 2010, no Sistema de Informação do Controle do Câncer de Mama (Sismama). Apesar de o Ministério da Saúde não recomendar o rastreamento mamográfico para mulheres com menos de 50 anos $^{12}$, optou-se por incluir as mulheres de 40 a 49 anos no estudo, uma vez que cerca de $40 \%$ das mamografias de rastreamento são realizadas nesse grupo etário $^{19}$. Decidiu-se incluir apenas mulheres reastreadas no segundo semestre de 2010, um ano após a implantação do sistema, para minimizar os problemas de registro de dados da fase inicial de implantação do sistema de informação.

Os dados das participantes foram relacionados, de forma probabilística, por meio do Programa Reclink ${ }^{20}$, conforme descrito por Tomazelli ${ }^{21,22}$, com as bases de dados dos Sistemas de Informação Hospitalar (SIH-SUS), Ambulatorial (SIA-SUS) e de Mortalidade (SIM) para se obterem informaçôes sobre a realização de biópsia, tratamento para câncer de mama e óbito até dezembro de 2012.

Foram selecionadas para o estudo no Sismama as variáveis: idade, raça/cor, repetição da mamografia, ter sido submetida a exame clínico das mamas (ECM) anteriormente, tamanho do nódulo, tipo de unidade que solicitou a mamografia, tipo de hospital que fez o tratamento, tipo de tratamento, estadiamento, AP das unidades responsáveis pela solicitação da mamografia, das unidades que realizaram a mamografia e das unidades de tratamento. A informação de biópsia foi identificada no próprio Sismama (módulo histopatológico) ou no SIA-SUS. O tipo de tratamento foi proveniente do SIH (cirurgias) ou do SIA - Autorizaçóes de Procedimento de Alta Complexidade em Oncologia (Apac-Oncologia) - e o estadiamento da Apac-Oncologia ${ }^{21,22}$. As variáveis utilizadas foram as disponíveis nas bases utilizadas e identificadas como aquelas que poderiam apresentar possível relação com o tempo de início de tratamento.

As classificaçôes do tipo de unidade que solicitou a mamografia e onde foi feito o tratamento partiram da identificação do perfil dessas unidades no Cadastro Nacional de Estabelecimentos de Saúde (Cnes) e da 
Portaria que habilitava os hospitais em estabelecimentos especializados para o tratamento do câncer no período estudado $^{23}$. As variáveis relativas às AP foram criadas a partir da identificação da localização dessas unidades.

A idade foi categorizada nas faixas de 40-49, 50-59, 60-69 anos. O tamanho do nódulo foi categorizado em menor que $21 \mathrm{~mm}$ e maior ou igual a $21 \mathrm{~mm}$. O tipo de unidade que solicitou a mamografia foi separado em unidade básica de saúde (UBS), unidade secundária, hospital geral e hospital especializado. O tipo de hospital que fez o tratamento (geral ou especializado) e o tipo de tratamento foram provenientes do SIH ou da Apac-Oncologia. Hospital especializado refere-se ao habilitado para o tratamento do câncer, conforme estabelecido em Portaria vigente para o período do estudo ${ }^{23}$.

Foram estudadas de forma dicotomizada: ter ECM, repetição da mamografia (nenhuma, uma vez ou mais) após o resultado alterado, e ter informação de biópsia.

A variável estadiamento foi inicialmente definida como in situ (0), precoce (I e II) e avançado (III e IV), e ficou restrita às mulheres que realizaram tratamento de quimioterapia (QT), hormonioterapia (HT) ou radioterapia (RT), tendo sido resgatada da Apac-Oncologia, mesmo que o tratamento tenha sido posterior à cirurgia.

A variável raça/cor, que estava ausente em $100 \%$ dos campos do Sismama na coorte estudada, foi recuperada por meio dos demais Sistemas de Informaçóes em Saúde (SIS), não sendo possível recuperá-la para oito mulheres. Utilizaram-se os seguintes critérios quando estava presente em mais de um SIS e eram discordantes: entre branca, parda e amarela, adotou-se a categoria branca; entre preta e parda, adotou-se não branca.

Inicialmente, foi feita análise descritiva das características das mulheres e calculados os tempos médio e mediano entre a data de resultado da mamografia alterada e o tempo de início do tratamento por AP. Verificou-se a distribuiçấo dos casos por estadiamento e por tipo de unidade que solicitou a mamografia.

Para a estimativa dos tempos entre o resultado da mamografia e o início do tratamento, utilizou-se o método Kaplan-Meier. Para verificar possíveis diferenças entre os estratos das variáveis selecionadas, utilizou-se teste Log-rank. O tipo de unidade que solicitou a mamografia foi estratificado em três categorias: UBS, unidade secundária ou hospital geral e hospital especializado; o tamanho do nódulo foi categorizado em não palpável $(<21 \mathrm{~mm})$ e palpável $(\geq 21 \mathrm{~mm})$.

O tempo em risco começou a partir da data de resultado da mamografia alterada (BI-RADS ${ }^{\ominus} 4$ ou 5) e terminou na data em que recebeu o primeiro tratamento para câncer de mama (cirurgia, QT/HT ou RT) ou na data de óbito ou na data em que o seguimento do presente estudo foi interrompido (censura administrativa em 31/12/2012). Considerou-se, como evento, a data de início do primeiro tratamento registrado no SIH ou na Apac-Oncologia das mulheres encontradas nesses sistemas. A opção de realizar o estudo até dezembro de 2012 levou em conta a substituição do Sismama por outro sistema de informação ${ }^{24}$.

Para avaliar os fatores associados ao tempo até o início do tratamento, utilizou-se o modelo de riscos proporcionais de Cox. Foram calculadas as hazard ratios (HR) brutas e ajustadas e os respectivos intervalos de confiança de 95\% (IC 95\%). Na análise múltipla, foram incluídas as variáveis selecionadas: $\mathrm{ECM}$, tipo de unidade solicitante da mamografia, repetição da mamografia, realização de biópsia e o tamanho do nódulo, ajustadas pela idade de forma contínua. O pressuposto de proporcionalidade do risco no tempo nas análises realizadas foi aferido a partir dos resíduos padronizados de Schoenfeld ${ }^{25}$.

Como nessa etapa houve quebra da proporcionalidade com a inclusão do variável tipo de unidade solicitante da mamografia, essa análise foi repetida com exclusão dessa variável.

Uma terceira análise foi feita incluindo-se a variável estadiamento categorizada em dois grupos (in situ + precoce e avançado), pois, como essa informação só está disponível na Apac-Oncologia, a sua introdução no modelo de análise leva ao viés diferencial, já que as mulheres que fizeram apenas cirurgia - e, por isso, só aparecem no SIH cuja base de dados náo registra o estadiamento - são aquelas que devem ter doença em estádios iniciais.

Foi realizada, ainda, uma análise adicional incluindo os cinco óbitos das mulheres, que ocorreram na coorte no período estudado. Nessa análise, para o cálculo das curvas de Kaplan-Meier, os óbitos foram tratados como perda de seguimento e considerou-se como data da censura a data do óbito. Foi utilizado também o modelo de riscos proporcionais de Cox, incluindo as mesmas variáveis selecionadas para o modelo anterior. Todas as análises estatísticas foram realizadas no programa $\mathrm{R}$ versão 3.1.126.

A pesquisa foi aprovada pelos Comitês de Ética em Pesquisa do Instituto de Medicina Social (IMS), Universidade do Estado do Rio de Janeiro (Uerj) (Parecer 1.105.945), Secretaria Municipal de Saúde (SMS) do Rio de Janeiro (Parecer 1.162.544) e do Instituto Nacional de Câncer José Alencar Gomes da Silva - INCA (Parecer 1.139.738).

\section{RESULTADOS}

Foram informadas no Sismama 12.183 mamografias de rastreamento de mulheres residentes no município 
do Rio de Janeiro no segundo semestre de 2010, sendo 10.330 mamografias $(84,4 \%)$ no grupo etário de 40-69 anos. Entre estas, foram identificadas 158 mulheres com suspeita de câncer de mama (BI-RADS 4 ou 5), 45 com idade de 40-49 anos e 113 com idade de 50-69 anos. O relacionamento dos registros dessas 158 mulheres com as bases SIA-SUS, SIH-SUS e SIM revelou que, para 67 $(42,4 \%)$, havia informação sobre o tratamento nas bases analisadas. Das 91 sem registro de tratamento, 86 não foram localizadas em qualquer outro registro e cinco foram localizadas no SIM. Das 67 mulheres tratadas, 66 tiveram câncer de mama e uma foi tratada por doença benigna. $\mathrm{O}$ resultado da mamografia de rastreamento dessas pacientes mostrou que 43 tiveram BI-RADS 4 e 23 BI-RADS $^{\circ} 5$.

$A$ análise a seguir considerou o total de mulheres com diagnóstico confirmado de câncer de mama. Entre estas, a média de idade foi de 55,8 anos (desvio-padrão $=7,2$ anos) e a mediana foi de 56 anos $\left(1^{\circ}\right.$ quartil $=50$ e $3^{\circ}$ quartil $=$ 62); 80,3\% tinham idade entre 50-69 anos e 47,0\% eram brancas. Entre as informaçóes registradas nos SIS, foi visto que, para 86,4\%, havia menção de ECM e, em 75,8\% dos casos, não havia nódulo palpável. Não havia informação sobre realização de biópsia no SUS para a maioria das pacientes $(87,9 \%)$ e, para $24,2 \%$, houve repetiçáo da mamografia. Metade das solicitaçóes de mamografia foi feita em hospital especializado. Na AP 2.2, se concentrou a maior parte das solicitaçôes e realizaçôes de mamografia (53,0\% e 75,8\%, respectivamente) (Tabela 1$)$.

A maior parte das mulheres $(93,9 \%)$ foi tratada em hospital especializado. A cirurgia foi o tratamento mais frequente $(43,9 \%)$ e a AP 2.2 , novamente, foi a que recebeu maior número de mulheres para tratamento $(63,6 \%)$. Entre os casos com estadiamento informado, $57,9 \%$ eram in situ ou precoce (Tabela 1 ).

O tempo médio entre uma mamografia alterada e o início do primeiro tratamento para o câncer de mama foi de 258 dias e o mediano de 206 dias, variando de 19 a 707 dias (Figura 1); o tempo médio foi superior a seis meses em todas as AP onde foi realizado tratamento.

Todas as mamografias solicitadas pela AP 2.2 eram de hospitais especializados. Nenhuma das mulheres em tratamento tinha mamografia solicitada por UBS das AP 2.1, 2.2 e 4.0. O estadiamento por tipo de unidade solicitante mostra que os estádios avançados foram menores nos hospitais especializados e gerais comparados às unidades secundárias $(52,9 \%)$ e UBS $(50,0 \%)$; foram excluídos nove casos sem informação de estadiamento (dados náo apresentados).

$\mathrm{Na}$ análise estratificada no modelo de Kaplan-Meier, apresentaram diferença estatisticamente significativa o grupo etário, a repetiçáo da mamografia e o tipo de unidade que solicitou a mamografia (Log-rank $<0,05)$
Tabela 1. Distribuição das características de mulheres com resultado suspeito e altamente suspeito de malignidade na mamografia de rastreamento (BI-RADS $® 4$ ou 5) e malignidade confirmada, município do Rio de Janeiro, 2010-2012

\begin{tabular}{l|c|c}
\multicolumn{1}{c|}{ Características estudadas } & N & $\%$ \\
\hline Grupo etário & 13 & 19,7 \\
\hline 40 a 49 anos & 27 & 40,9 \\
\hline 50 a 59 anos & 26 & 39,4 \\
\hline 60 a 69 anos & & \\
\hline Raça/cor & 31 & 47,0 \\
\hline Branca & 27 & 40,9 \\
\hline Não Branca & 8 & 12,1 \\
\hline Missing
\end{tabular}

Tipo de unidade solicitante da mamografia

\begin{tabular}{l|c|c}
\hline Unidade Básica de Saúde & 10 & 15,2 \\
\hline Unidade secundária & 18 & 27,3 \\
\hline Hospital especializado & 33 & 50,0 \\
\hline Hospital geral & 5 & 7,6 \\
\hline
\end{tabular}

Exame clínico das mamas anteriormente

\begin{tabular}{l|c|c}
\hline Não & 9 & 13,6 \\
\hline Sim & 57 & 86,4 \\
\hline
\end{tabular}

Repetição da mamografia

\begin{tabular}{l|l|l}
\hline Nenhuma & 50 & 75,8 \\
\hline 1 ou mais vezes & 16 & 24,2 \\
\hline
\end{tabular}

Informação de biópsia

\begin{tabular}{l|c|c}
\hline Não & 58 & 87,9 \\
\hline Sim & 8 & 12,1 \\
\hline
\end{tabular}

Tamanho do nódulo

\begin{tabular}{l|l|l}
\hline$<21 \mathrm{~mm}^{1}$ & 50 & 75,8 \\
\hline$>21 \mathrm{~mm}$ & 16 & 24,2 \\
\hline
\end{tabular}

Área Programática da unidade que solicitou mamografia

\begin{tabular}{l|c|c}
\hline 1.0 & 19 & 28,8 \\
\hline 2.1 & 1 & 1,5 \\
\hline 2.2 & 35 & 53,0 \\
\hline 3.1 & 3 & 4,5 \\
\hline 3.2 & 3 & 4,5 \\
\hline 3.3 & 2 & 3,0 \\
\hline 5.2 & 2 & 3,0 \\
\hline 5.3 & 1 & 1,5 \\
\hline
\end{tabular}

Área Programática da unidade que realizou a mamografia

\begin{tabular}{c|c|c}
\hline 2.2 & 50 & 75,8 \\
\hline 2.3 & 10 & 15,2 \\
\hline 3.2 & 1 & 1,5 \\
\hline 4.0 & 5 & 7,6 \\
\hline
\end{tabular}


Tabela 1. continuação

\begin{tabular}{l|c|c}
\hline \multicolumn{1}{c|}{ Características estudadas } & $\mathbf{N}$ & $\%$ \\
\hline \multicolumn{1}{l}{ Área Programática da unidade que tratou } \\
\hline 1.0 & 10 & 15,2 \\
\hline 2.1 & 1 & 1,5 \\
\hline 2.2 & 42 & 63,6 \\
\hline 3.1 & 9 & 13,6 \\
\hline 3.2 & 3 & 4,5 \\
\hline 4.0 & 1 & 1,5 \\
\hline Estadiamento & 2 & 3,0 \\
\hline In situ & 31 & 47,0 \\
\hline Precoce & 24 & 36,4 \\
\hline Avançado & 9 & 13,6 \\
\hline Missing & 62 & 93,9 \\
\hline Tipo de unidade que fez o tratamento \\
\hline Hospital Especializado & 4 & 6,1 \\
\hline Hospital Geral & 29 & 43,9 \\
\hline \multicolumn{3}{|l|}{ Tipo de tratamento } \\
\hline Procedimentos Cirúrgicos & 19 & 28,8 \\
\hline Quimioterapia & 7 & 10,6 \\
\hline Hormonioterapia & 11 & 16,7 \\
\hline Radioterapia
\end{tabular}

Nota: ${ }^{\text {incluídas }} 31$ mulheres sem informação de presença de nódulos.

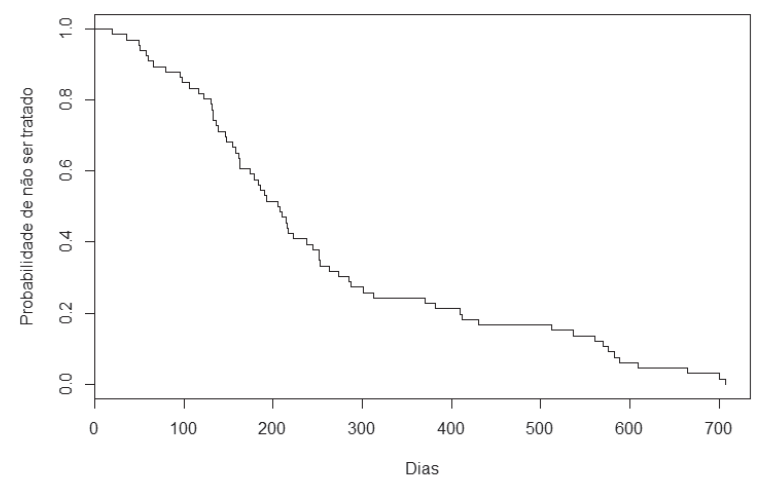

Figura 1. Tempo entre resultado de mamografia de rastreamento com lesão suspeita ou altamente suspeita de malignidade (BI-RADS ${ }^{\circledR} 4$ ou 5) e início de tratamento para mulheres rastreadas para o câncer de mama no segundo semestre de 2010, município do Rio de Janeiro, 2010-2012

(Figura 2). O tempo mediano para início do tratamento foi menor para mulheres que tiveram mamografia solicitada por hospital especializado (138 dias), que referiram ECM anterior (205 dias), que não repetiram a mamografia (184 dias) e que não tinham informação de biópsia (199 dias). 2a)

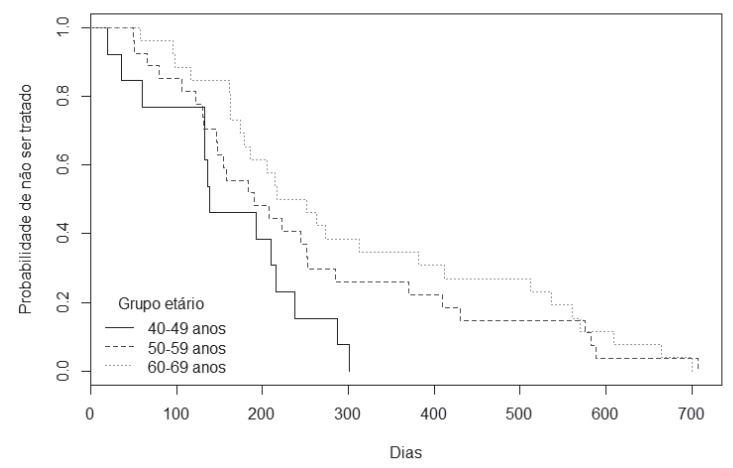

Log-rank $\mathrm{p}=0,043$

b)

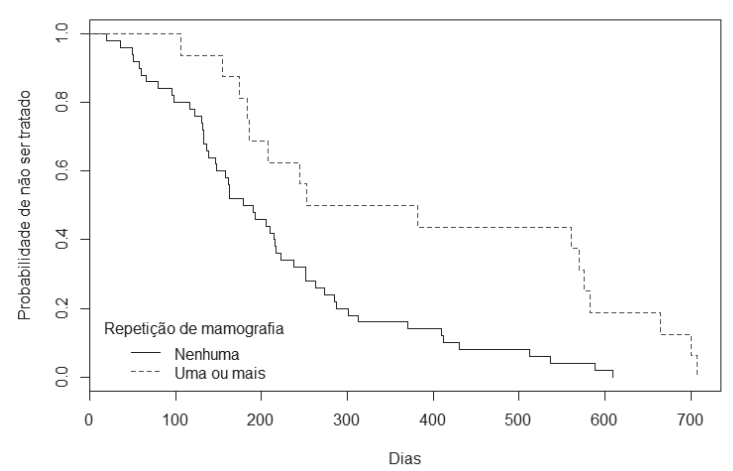

Log-rank $p=0,002$

2c)

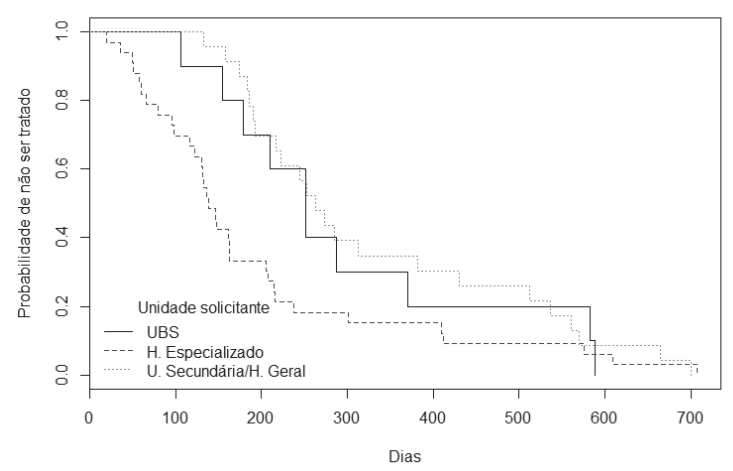

Log-rank $p=0,039$

Figura 2. Tempo entre resultado da mamografia com lesão suspeita ou altamente suspeita de malignidade e início de tratamento entre mulheres rastreadas para o câncer de mama no segundo semestre de 2010 segundo grupo etário (2a), repetição de mamografia (2b) e tipo de unidade solicitante (2c), município do Rio de Janeiro, 2010-2012

No modelo múltiplo de Cox em que foram incluídas as variáveis ECM, tipo de unidade solicitante da mamografia, repetição da mamografia, realização de biópsia e o tamanho do nódulo, a repetiçáo de mamografia (uma vez ou mais) foi a única variável que se associou a um maior tempo de espera para o tratamento $(0,39$; IC $95 \%$ : 0,20-0,78), porém o resíduo padronizado de Schoenfeld do modelo indicou quebra da proporcionalidade do risco no tempo (Tabela 2). A avaliação dos riscos mostrou que a quebra da proporcionalidade era atribuída à variável tipo de unidade solicitante da mamografia. 
Tabela 2. Tempo mediano e hazard ratio para o tempo entre mamografia suspeita ou altamente suspeita de malignidade e início do tratamento associado a variáveis selecionadas em mulheres rastreadas no município do Rio de Janeiro, 2010-2012

\begin{tabular}{|c|c|c|}
\hline $\begin{array}{l}\text { Características } \\
\text { estudadas }\end{array}$ & $\begin{array}{c}\text { Tempo } \\
\text { mediano } \\
\text { (dias) }\end{array}$ & $\begin{array}{l}\text { Modelo final } \\
\left(\text { HR }^{\mathrm{a}} \text { e } I_{95 \%}\right)\end{array}$ \\
\hline \multicolumn{3}{|c|}{ Exame clínico das mamas anteriormente } \\
\hline Não & 263 & 1 \\
\hline Sim & 205 & $1,20(0,50-2,88)$ \\
\hline \multicolumn{3}{|c|}{ Tipo de unidade solicitante da mamografia } \\
\hline $\begin{array}{l}\text { Unidade Básica de } \\
\text { Saúde }\end{array}$ & 251 & 1 \\
\hline $\begin{array}{l}\text { Unidade secundária/ } \\
\text { geral }\end{array}$ & 263 & $1,63(0,65-4,07)$ \\
\hline Hospital especializado & 138 & $2,62(0,98-6,99)$ \\
\hline \multicolumn{3}{|c|}{ Repetição mamografia } \\
\hline Nenhuma & 184 & 1 \\
\hline 1 ou mais vezes & 317 & $0,39(0,20-0,78)$ \\
\hline \multicolumn{3}{|l|}{ Tamanho do nódulo } \\
\hline Não palpável & 195 & 1 \\
\hline Palpável & 251 & $0,81(0,43-1,51)$ \\
\hline \multicolumn{3}{|l|}{ Informação de biópsia } \\
\hline Não & 199 & 1 \\
\hline Sim & 230 & $0,50(0,20-1,29)$ \\
\hline
\end{tabular}

Notas: a Ajustado por idade e por todas as variáveis da tabela; Teste resíduo Schoenfeld: $(\mathrm{p}<0,05)$.

$\mathrm{Na}$ análise do modelo, excluindo o tipo de unidade solicitante da mamografia, a repetiçáo de mamografia (uma vez ou mais) foi a única variável que se associou a um maior tempo de espera para o tratamento $(0,36$; IC $95 \%$ : 0,19-0,72). Ter realizado ECM anteriormente, embora tenha alcançado $\mathrm{HR}$ de 1,40 , não obteve intervalo de confiança estatisticamente significativo e demasiadamente amplo, o que torna a estimativa muito imprecisa (Tabela 3). As hazard ratios do modelo com a variável tipo de unidade solicitante da mamografia não diferiram muito do modelo sem essa variável.

A análise dos resíduos padronizados de Schoenfeld mostrou que não houve violação do pressuposto de proporcionalidade dos riscos no tempo $(\mathrm{p}=0,571)$ neste último modelo.

$\mathrm{Na}$ análise em separado, incluindo a variável estadiamento, o tempo médio para tratamento foi de 262 dias, com mediana de 214 dias (variando de 19-700 dias). As curvas de Kaplan-Meier para estadiamento não mostraram diferença (Log-rank=0,747) e a regressão de riscos proporcionais de Cox não diferiu da análise anterior.

$\mathrm{Na}$ análise com inclusão dos cinco óbitos, o tempo médio para tratamento foi de 262 dias, o que aumentaria em quatro dias o tempo médio encontrado, quando esses
Tabela 3. Tempo mediano e hazard ratio para o tempo entre mamografia suspeita ou altamente suspeita de malignidade e início do tratamento associado às variáveis descritas em mulheres rastreadas no município do Rio de Janeiro, 2010-2012

\begin{tabular}{l|c|c}
\hline $\begin{array}{c}\text { Características } \\
\text { estudadas }\end{array}$ & $\begin{array}{c}\text { Tempo } \\
\text { mediano } \\
\text { (dias) }\end{array}$ & $\begin{array}{c}\text { Modelo final } \\
\left.\text { (HRa } \text { IC I }_{95 \%}\right)\end{array}$ \\
\hline Exame clínico das mamas anteriormente \\
\hline Não & 263 & 1 \\
\hline Sim & 205 & $1,40(0,67-2,91)$ \\
\hline Repetição mamografia \\
\hline Nenhuma & 184 & 1 \\
\hline 1 ou mais vezes & 317 & $0,36(0,19-0,72)$ \\
\hline Tamanho do nódulo \\
\hline Não palpável & 195 & 1 \\
\hline Palpável & 251 & $0,80(0,44-1,45)$ \\
\hline Informação de biópsia \\
\hline Não & 199 & 1 \\
\hline Sim & 230 & $0,86(0,39-1,90)$ \\
\hline
\end{tabular}

Notas: ${ }^{2} A j u s t a d o$ por idade e por todas as variáveis da tabela; Teste resíduo Schoenfeld: $(\mathrm{p}<0,05)$.

casos não foram incluídos na coorte. A análise estratificada no modelo Kaplan-Meier manteve com diferença significativa a repetição da mamografia e o tipo de unidade que solicitou a mamografia. O modelo múltiplo de riscos proporcionais de Cox manteve-se similar ao modelo sem inclusão dos óbitos e na análise dos resíduos de Schoenfeld, o pressuposto de proporcionalidade dos riscos no tempo permaneceu não violado $(\mathrm{p}=0,421)-$ dados náo apresentados.

\section{DISCUSSÃO}

A base de referência para a população deste estudo partiu de um total de 10.330 mamografias de rastreamento em mulheres de 40 a 69 anos registradas no Sismama no segundo semestre de 2010. Destas, foram identificadas 158 mulheres com BI-RADS ${ }^{\circledast} 4$ ou 5 . Utilizando, nesse grupo, o parâmetro de programação que estima que 2,2\% do total de mamografias de rastreamento de mulheres na faixa etária de 50-69 anos demandariam investigação diagnóstica ${ }^{27}$, seriam esperados 228 casos alterados (68 no grupo etário de 40-49 anos e 160 no de 50-69 anos). O número de casos encontrados (158, 45 entre 40-49 anos e 113 entre 50-69 anos) foi inferior ao estimado. Como o parâmetro baseou-se no parâmetro canadense, onde a incidência do câncer de mama é maior do que no Brasil, e como este foi estabelecido para indivíduos e o Sismama registra exames e não mulheres, é possível que a diferença real seja menor do que a encontrada (70 casos). Cabe destacar que os parâmetros nacionais utilizados foram 
construídos considerando as referências de outro país. Tendo em vista a proporção estimada de câncer de mama, para lesōes não palpáveis, de um resultado BI-RADS 4 como $20 \%$ e do BI-RADS $^{\circ} 5$ como $80 \%{ }^{28}$, seriam esperados 52 casos de câncer de mama na faixa etária de 40-69 anos, inferior ao número incluído na coorte (66 mulheres).

Ressalta-se que o parâmetro aplicado $(2,2 \%)$ incluiu também mulheres de 40-49 anos que tinham mamografia de rastreamento, já que não existe parâmetro para esse grupo etário. A utilização do parâmetro nesse segmento pode ter elevado o número de casos que necessitariam de investigação histopatológica.

Os principais achados deste estudo foram: (i) longo tempo entre o resultado da mamografia alterada e início do tratamento - sendo menor em mulheres mais jovens; (ii) tempo mediano para início do tratamento menor para mamografia de rastreamento solicitada por hospital especializado; e (iii) tempo maior quando houve repetição da mamografia.

Destacam-se a baixa proporçáo de mamografias alteradas solicitadas pelas UBS e a ausência destas nas AP 2.1, 2.2 e 4.0. O tempo para tratamento foi menor, ainda que elevado, quando a mamografia foi solicitada por hospitais especializados; e maior, quando solicitada por unidades secundária/hospital geral. Apesar do prazo de até 60 dias para tratamento de câncer, contados a partir do diagnóstico histopatológico ${ }^{18}$, não existe normativa ou parâmetro estabelecido quanto ao tempo de espera entre um exame de rastreamento que demande investigação diagnóstica e tratamento. Possuir diagnóstico prévio ao chegar no hospital especializado influência o tempo para início do tratamento do câncer de mama ${ }^{29}$. O Programa de rastreamento canadense estabelece a meta de que 90\% ou mais das pacientes tenham o diagnóstico efetuado até cinco semanas do exame alterado de rastreamento para aquelas que confirmaram o diagnóstico com punção por agulha fina. Para aquelas que realizaram biópsia tecidual (core biopsy ou biópsia cirúrgica), esse prazo pode ser estendido para até sete semanas ${ }^{14}$. Os programas do Reino Unido e da Inglaterra esperam que $90 \%$ ou mais das mulheres sejam admitidas para tratamento dentro de dois meses desde a data do início da investigação diagnóstica ${ }^{15,16,30}$. Nos Estados Unidos, das mulheres que usam o Programa Nacional de Detecçáo Precoce do câncer de Mama e do Colo do Útero (NBCCEDP), 80\% foram diagnosticadas em 60 dias a partir de uma mamografia de rastreamento alterada e $94 \%$ iniciaram o tratamento em até 60 dias após o diagnóstico ${ }^{31}$.

$\mathrm{O}$ fato de as mulheres com solicitação de mamografia de rastreamento por hospitais especializados apresentarem tempo menor para tratamento quando comparadas com aquelas com exame solicitado por UBS, sugere que parte dessas mulheres deva ter sido encaminhada para esses hospitais após uma mamografia prévia suspeita não identificada nas bases de dados do estudo. Estudo em um hospital especializado no município do Rio de Janeiro ${ }^{32}$ identificou que, entre as mulheres que levaram o exame mamográfico na consulta, $68 \%$ o tinham realizado em serviços privados e apenas $35,6 \%$ das mulheres tinham diagnóstico histopatológico, dos quais $67,6 \%$ foram realizados em serviços privados. Outras possíveis explicaçôes são: (i) mulheres que realizaram mamografia suspeita em datas anteriores ao período de início deste estudo; (ii) mulheres que tiveram mamografia prévia com resultado alterado fora da rede SUS; ou (iii) que tiveram câncer de mama no passado e estavam em acompanhamento no hospital especializado, porém sem que essa informação fosse registrada ou identificada nos sistemas de informaçôes assistenciais do SUS no período do estudo.

Não foi possível determinar no presente estudo o motivo pelo qual náo foram localizadas informaçóes de investigação histopatológica para a maioria (87,9\%) das mulheres. É possível que esses exames tenham sido realizados na rede privada ou fora do município do Rio de Janeiro, bem como náo terem sido localizados nos SIS ou não terem sido realizadas as biópsias. Os poucos casos identificados com registro de investigação diagnóstica sinalizam dificuldade para a realização de biópsia nos serviços da rede pública de saúde, o que é consistente com resultados de outros estudos ${ }^{32-34} \mathrm{e}$ sugere que a busca pelo exame ocasione demora para iniciar o tratamento. Também não foi possível explorar o fato de as mulheres, que apresentaram resultado de mamografia com BI-RADS ${ }^{\ominus} 4$ ou 5 , terem repetido a mamografia, já que a conduta nesses casos é prosseguir para a investigação histopatológica ${ }^{13}$.

Observou-se que uma elevada proporção de mulheres em tratamento foi submetida à ECM. Apesar de as atuais diretrizes para deteç̧ão precoce do câncer de mama no Brasil $^{12}$ não recomendarem o ECM como estratégia de detecção precoce do câncer de mama, este fazia parte das recomendaçôes de rastreamento anteriores. Esse achado pode indicar que, durante os anos estudados, houve uma maior adesão a essa prática pelos profissionais. Contudo, quase um quarto das mamografias de rastreamento apresentava nódulos palpáveis, o que, diante da elevada proporção de realização do ECM, sugere uma solicitação equivocada da mamografia indicada como "rastreamento", quando o correto seria "diagnóstica". Deve-se considerar, porém, que, ter nódulo palpável não resultou em ter um menor tempo entre a mamografia e o início do tratamento.

Destaque deve ser dado ainda ao fato de que a única condição que se associou a um maior tempo de espera 
entre o rastreamento e o início do tratamento foi a repetição da mamografia.

Embora seja expressivo o número de hospitais especializados que solicitaram mamografia de rastreamento, alguns deles realizam também atendimento clínico para a comunidade, de forma que as mamografias de rastreamento não são necessariamente provenientes de uma alteração prévia identificada na rede assistencial.

Como visto em outros estudos no país, metade dos cânceres de mama detectados são estádios in situ e precoce ${ }^{7,32}$. A maior proporçáa de mulheres com estádios III e IV, excluídas as perdas (missings), teve mamografia de rastreamento solicitada por unidade secundária ou hospital geral, sugerindo dificuldade de acesso à rede básica assistencial. Outra possível explicação seria que essas mulheres realizaram mamografia fora da rede e levaram os resultados para essas unidades, que repetiram a mamografia pela baixa qualidade do exame anterior.

A falta de associação entre estadiamento e tempo de espera para tratamento deve ser vista com cautela dada a quantidade de informaçóes faltantes para essa variável. Tem sido relatado que as mulheres diagnosticadas em fase precoce da doença têm apresentado um maior tempo para confirmação diagnóstica ${ }^{32}$ e tratamento ${ }^{29}$.

O número pequeno de mulheres que compôs a coorte foi a principal limitação do estudo, ainda que se tenha partido de todas as mamografias alteradas $\left(\mathrm{BI}^{-\mathrm{RADS}^{\circ}}\right.$ 4 ou 5) registradas na rede SUS no segundo semestre de 2010, o que correspondeu a 206 mulheres. Desse total, 86 , com idade entre 40 e 69 anos, não foram localizadas e é possível que parte dessas tenha realizado a biópsia e o tratamento, se necessário, em serviços privados. Essa perda no seguimento poderia, potencialmente, introduzir um viés de seleção no estudo, no sentido de que mulheres não encontradas nas bases de dados dos sistemas, que foram relacionados ao Sismama, teriam um risco aumentado ou diminuído de maior tempo de espera para o tratamento. No entanto, a comparação feita entre o grupo de mulheres com informaçáo sobre tratamento e aquelas não encontradas nas bases com informaçóes sobre tratamento náo mostrou diferenças entre as características de idade, exame clínico da mama anterior, tipo de unidade solicitante da mamografia, AP de solicitação e de realização da mamografia $a^{34}$. Esse problema, contudo, contribuiu para que o número de mulheres seguidas não fosse suficiente para assegurar precisão aos resultados encontrados e, por isso, algumas estimativas podem não ter alcançado significância estatística, mas podem indicar o que seria esperado se amostra estudada fosse ampliada.

Outro fator limitante foi a qualidade dos dados das bases secundárias decorrentes da não obrigatoriedade de preenchimento de alguns campos, a ausência de sistemas de crítica que permitam validar a informação durante o seu registro, a falta de padronização das variáveis entre os sistemas e o não preenchimento de alguns campos como o de raça/cor.

Ainda como limitação, deve ser mencionado que o desenho de relacionamento das bases de dados que gerou a coorte não previu o relacionamento com mamografias de um período anterior, de forma a excluir mamografias prévias com resultado alterado ${ }^{21}$. Esse fato pode explicar a elevada proporção de mamografias de rastreamento solicitadas por hospital especializado: talvez parte das mulheres que tiveram tais solicitações já tivesse uma mamografia anterior alterada, e a mamografia detectada no estudo fosse uma repetiçáo no hospital especializado.

\section{CONCLUSÃO}

O tempo entre o resultado da mamografia alterada e o início do tratamento foi considerado longo, sendo o tempo mediano de 206 dias (6,8 meses).

Os resultados encontrados trouxeram algumas questóes que merecem ser melhor investigadas, como: o motivo de repetição da mamografia alterada; o pequeno número de biópsias registradas; o tempo para início de tratamento ser superior a seis meses mesmo quando as mamografias são solicitadas por hospitais especializados e o motivo pelo qual as mamografias alteradas foram pouco identificadas em UBS. O pequeno número de mulheres acompanhadas não permite que esses achados possam ser extrapolados para a população de mulheres rastreadas para o câncer de mama no município do Rio de Janeiro, mas servem, contudo, de alerta para que medidas possam ser tomadas no sentido de permitir o monitoramento das açôes de controle do câncer de mama e o aprimoramento dos Sistemas de Informação do SUS.

\section{CONTRIBUIÇÕES}

Jeane Glaucia Tomazelli contribuiu com a concepção e delineamento do estudo, análise e interpretação dos dados, redação e revisão crítica relevante do conteúdo intelectual do manuscrito. Isabel dos-Santos-Silva e Gulnar Azevedo e Silva contribuíram na análise e interpretação dos dados e revisão crítica do artigo. Todos os autores aprovaram a versão final do manuscrito e declaram ser responsáveis por todos os aspectos do trabalho, garantindo sua precisão e integridade.

\section{DECLARAÇÃO DE CONFLITO DE INTERESSES}

Nada a declarar. 


\section{FONTES DE FINANCIAMENTO}

Não há.

\section{REFERÊNCIAS}

1. Instituto Nacional de Câncer José de Alencar Gomes da Silva. Estimativa 2016: incidência de câncer no Brasil. Rio de Janeiro: INCA; 2015. 122 p.

2. Instituto Nacional de Câncer. A situação do câncer no Brasil. Rio de Janeiro: INCA; 2006. 119 p.

3. Instituto Nacional de Câncer José de Alencar Gomes da Silva. Atlas on-line de Mortalidade [Internet]. Rio de Janeiro: INCA; c1996-2014. [acesso 2018 Abr 6]. Disponível em: https://www.inca.gov.br/app/ mortalidade.

4. Girianelli VR, Gamarra CJ, Silva GA. Os grandes contrastes na mortalidade por câncer do colo uterino e de mama no Brasil. Rev Saude Pública. 2014;48(3):459-67. doi: http://dx.doi.org/10.1590/ S0034-8910.2014048005214.

5. Lee BL, Liedke PE, Barrios CH, Simon SD, Finkelstein DM, Goss PE. Breast cancer in Brazil: present status and future goals. Lancet Oncol. 2012;13(3):e95-e102. doi: http://dx.doi.org/10.1016/S1470-2045(11)70323-0.

6. Instituto Nacional de Câncer José de Alencar Gomes da Silva. Controle dos cânceres do colo do útero e da mama. 2. ed. Brasília: Ministério da Saúde; 2013. 124 p. (Cadernos de Atenção Básica, n. 13).

7. Thuler LCS, Mendonça GA. Estadiamento inicial dos casos de câncer de mama e colo do útero em mulheres brasileiras. Rev Bras Ginecol Obstet. 2005;27(11):656-60. doi: http://dx.doi.org/10.1590/ S0100-72032005001100004.

8. Gebrim LH, Quadros LGA. Rastreamento do câncer de mama no Brasil. Rev Bras Ginecol Obstet. 2006;28(6):319-23. doi: http://dx.doi.org/10.1590/ S0100-72032006000600001.

9. Ministério da Saúde (BR). Secretaria de Gestão Participativa. Reorganizando o SUS no Município do Rio de Janeiro. Brasília, DF: Ministério da Saúde; 2005. 118 p. (Série D. Reunióes e Conferências).

10. Prefeitura da Cidade do Rio de Janeiro. Secretaria Municipal de Saúde e Defesa Civil. Plano Municipal de Saúde do Rio de Janeiro: PMS 2010 - 2013 [Internet]. Rio de Janeiro: Prefeitura do Rio; 2009. [acesso 2015 Ago 04]. Disponível em: http://www. rio.rj.gov.br/dlstatic/10112/3700816/4130215/ PLANOMUNICIPALDESAUDE20102013.pdf.

11. Secretaria Municipal de Saúde do Rio de Janeiro. Subsecretaria de Açôes e Serviços de Saúde. SVS. Anexo Técnico I: informaçốes sobre todas as áreas de planejamento [Internet]. Rio de Janeiro: Prefeitura do Rio; [data desconhecida]. [acesso 2015 Ago 04]. Disponível em: http:/www.rio.rj.gov.br/dlstatic/10112/1529762/ DLFE-220205.pdf/1.0

12. Instituto Nacional de Câncer José Alencar Gomes da Silva. Diretrizes para a detecção precoce do câncer de mama no Brasil [Internet]. Rio de Janeiro: INCA; 2015. [acesso 2015 Jun 15]. Disponível em: https://www.inca. gov.br/sites/ufu.sti.inca.local/files//media/document// diretrizes_deteccao_precoce_cancer_mama_brasil.pdf

13. D'Orsi CJ, Sickles EA, Mendelson EB, Morris EA, et al. ACR BI-RADS ${ }^{\oplus}$ Atlas, Breast Imaging Reporting and Data System. Reston, VA, American College of Radiology; 2013.

14. Canadian Partnership Against Cancer. Report from the evaluation indicators working group: guidelines for monitoring breast cancer screening program performance. 3rd ed. Toronto: Canadian Partnership Against Cancer; 2013. 46 p.

15. Department of Health; NHS England. Public health functions to be exercised by NHS England: breast screening programme [Internet]. NHS England; 2013. [cited 2015 May 13]. Available from: https:// assets.publishing.service.gov.uk/government/uploads/ system/uploads/attachment_data/file/192975/24_ Breast_Screening_Programme_service_specification_ VARIATION_130422_-NA.pdf Service specification no 24.

16. The National Health Service Breast Screening Programme. Monitoring NHSBSP standards: a guide for quality assurance reference centres [Internet]. Version 3. Sheffield: NHS Cancer Screening Programmes; Dec 2005. [cited 2014 Mar 21]. Available from: www. cancerscreening.nhs.uk.

17. Perry N, Broeders M, Wolf C, Tömberg S, Holland R, von Karsa L. European guidelines for quality assurance in breast cancer screening and diagnosis. Fourth editionsummary document. Ann Oncol. 2008 Feb;19(4):61422. doi: https://doi.org/10.1093/annonc/mdm481.

18. Presidência da República (BR). Lei No 12.732, de 22 de novembro de 2012 [Internet]. [acesso 2015 Jun 01]. Disponível em: http://www.planalto.gov.br/ CCIVIL_03/_Ato2011-2014/2012/Lei/L12732.htm.

19. Instituto Nacional de Câncer José de Alencar Gomes da Silva. Avaliação de indicadores das açóes de detecção precoce dos cânceres do colo do útero e de mama - Brasil e Regiôes, 2013 [Internet]. Rio de Janeiro: INCA; 2015 Jan [acesso 2015 Jun 01]. 29 p. Disponível em: https:// www.inca.gov.br/sites/ufu.sti.inca.local/files//media/ document//avaliacao-indicadores-colo-e-mama-2013.pdf.

20. Camargo Jr KR, Coeli CM. RecLink III: relacionamento probabilístico de registros. Versão 3.1.6.3160. Rio de Janeiro: [editor desconhecido]; 2007.

21. Tomazelli JG, Girianelli VR, Azevedo e Silva G. Estratégias usadas no relacionamento entre Sistemas de Informaçōes em Saúde para seguimento das mulheres 
com mamografias suspeitas no Sistema Único de Saúde. Rev Bras Epidemiol. 2018;21:e180015. Epub 2018 Ago 27. doi: http://dx.doi.org/10.1590/1980549720180015.

22. Tomazelli JG, Girianelli, VR, Azevedo e Silva, G. Mulheres rastreadas para câncer de mama: acompanhamento por meio dos Sistemas de Informação em Saúde, 2010-2012. Epidemiol. Serv. Saude. 2018;21:E180015. doi: http:// dx.doi.org/10.1590/1980-549720180015.

23. Ministério da Saúde (BR), Secretaria de Atenção à Saúde. Portaria No 741, de 19 de dezembro de 2005 [Internet]. [acesso 2015 Jul 23]. Disponível em: http://bvsms.saude.gov.br/bvs/saudelegis/sas/2005/ prt0741_19_12_2005.html.

24. Ministério da Saúde (BR), Gabinete do Ministro. Portaria No 3.394, de 30 de desembro de 2013. [Internet]. [acesso 2015 Jul 23]. Disponível em: http://bvsms.saude.gov.br/ bvs/saudelegis/gm/2013/prt3394_30_12_2013.html.

25. Carvalho MS, Andreozzi VL, Codeço CT, Campos DP, Barbosa MTS, Shimakura SE. Análise de sobrevivência: teoria e aplicaçóes em saúde. 2. ed. Rio de Janeiro: Fiocruz; 2011. 432 p.

26. R Foundation for Statistical Computing. The R Project for Statistical Computing. [computer program]. R version 3.1.1. [Internet]. [place unknown]: The $\mathrm{R}$ foundation; 2014. [cited 2015 Jul 23]. Available from: https://www.R-project.org.

27. Instituto Nacional de Câncer. Parâmetros para o rastreamento do câncer de mama: recomendaçóes para gestores estaduais e municipais. Rio de Janeiro: INCA; 2009. 13 p.

28. Kestelman FP, Souza GA, Thuler LC, Martins G, Freitas VAR, Canella EO. Breast Imaging Reporting and Data System - BI-RADS ${ }^{\oplus}$ : valor preditivo positivo das categorias 3, 4 e 5. Revisão Sistemática da Literatura. Radiol Bras. 2007;40(3):173-7. doi: http://dx.doi. org/10.1590/S0100-39842007000300008.

29. Medeiros GC, Bergmann A, Aguiar SS, Thuler LCS. Análise dos determinantes que influenciam o tempo para o início do tratamento de mulheres com câncer de mama no Brasil. Cad Saúde Pública. 2015;31(6):1269-82. doi: http://dx.doi.org/10.1590/0102-311X00048514.

30. Health and Social Care Information Centre, Screening and Immunisations team. Breast Screening Programme, England 2011-12 [Internet]. London: NHS Information Centre; 2013. [cited 2014 Mar 21]. Available from: https://files.digital.nhs.uk/publicationimport/pub10xxx/ pub10339/bres-scre-prog-eng-2011-12-rep.pdf.

31. Richardson LC, Royalty J, Howe W, Helsel W, Kammerer W, Benard VB. Timeliness of breast cancer diagnosis and initiation of treatment in the National Breast and Cervical Cancer Early Detection Program, 1996-2005. Am J Public Health. 2010 Sep;100(9):1769-76. doi: http://dx.doi.org/10.2105/AJPH.2009.160184. Health and Social Care Information Centre, Screening and Immunisations team

32. Rezende MCR, Koch HA, Figueiredo JA, Thuler LCS. Causas do retardo na confirmação diagnóstica de lesôes mamárias em mulheres atendidas em um centro de referência do Sistema Único de Saúde no Rio de Janeiro. Rev Bras Ginecol Obstet. 2009;31(2):75-81. doi: http:// dx.doi.org/10.1590/S0100-72032009000200005.

33. Silva AG, Bustamante-Teixeira MT, Aquino EML, Tomazelli JG, Santos-Silva I. Acesso à detecção precoce do câncer de mama no Sistema Único de Saúde: uma análise a partir dos dados do Sistema de Informaçóes em Saúde. Cad Saúde Pública. 2014 Jul;30(7):1537-50. doi: http://dx.doi.org/10.1590/0102-311X00156513.

34 Tomazelli JG, Silva GA. Rastreamento do câncer de mama no Brasil: uma avaliação da oferta e utilização da rede assistencial do SUS no período 2010-2012. Epidemiol Serv Saude. 2017;26(4): 713-24. doi: http:// dx.doi.org/10.5123/S1679-49742017000400004.

Recebido em 29/9/2018 Aprovado em 7/1/2018 